University of Nebraska - Lincoln

DigitalCommons@University of Nebraska - Lincoln

April 1985

\title{
Random magnetism in amorphous rare-earth alloys (invited)
}

David J. Sellmyer

University of Nebraska-Lincoln, dsellmyer@unl.edu

S. Nafis

University of Nebraska - Lincoln

Follow this and additional works at: https://digitalcommons.unl.edu/physicssellmyer

Part of the Physics Commons

Sellmyer, David J. and Nafis, S., "Random magnetism in amorphous rare-earth alloys (invited)" (1985). David Sellmyer Publications. 148.

https://digitalcommons.unl.edu/physicssellmyer/148

This Article is brought to you for free and open access by the Research Papers in Physics and Astronomy at DigitalCommons@University of Nebraska - Lincoln. It has been accepted for inclusion in David Sellmyer Publications by an authorized administrator of DigitalCommons@University of Nebraska - Lincoln. 


\title{
Random magnetism in amorphous rare-earth alloys (unvited)
}

\author{
D. J. Sellmyer and S. Nafis \\ Behlen Laboratory of Physics, University of Nebraska, Lincoln, Nebraska 68588-0111
}

\begin{abstract}
Several aspects of the magnetic transitions seen in rare-earth metallic glasses are discussed, particularly with reference to recent theoretical work. These include: (a) apparent double transitions observed in $\mathrm{Gd}$ glasses where exchange fluctuations are important, (b) evidence for a correlated speromagnetic state recently predicted by Chudnovsky and Serota, and (c) the analysis of a $\mathrm{Tb}$ glass with strong random anisotropy in terms of an Ising-type spin-glass transition.
\end{abstract}

\section{INTRODUCTION}

Magnetic glasses based on the rare earths form an interesting laboratory system for studying phase transitions in the presence of random interactions. In these materials such interactions include random magnetic anisotropy (RMA), exchange fluctuations, and random atomic interactions which can induce chemical short-range order. Rare-earth glasses also are of great interest because of their potential as magnetic information storage media.

In this paper we shall briefly review recent theoretical advances in the RMA problem and present some experimental results that impinge upon this work. Our focus is upon the character of the magnetic transitions (whether glass transitions or phase transitions) and upon their relationship to analogous ones in canonical spin glasses such as $\mathrm{Cu}: \mathrm{Mn}$.

\section{RECENT THEORETICAL DEVELOPMENTS}

To provide a framework for the discussion of magnetic properties of rare-earth glasses, consider the model Hamiltonian

$$
H=-\sum_{i, j}^{\prime}\left(J_{0}+\Delta J_{i j}\right) \mathbf{J}_{i}^{\prime} \cdot \mathbf{J}_{j}^{\prime}-\sum_{i} D\left(\hat{n}_{i} \cdot \mathbf{J}_{i}^{\prime}\right)^{2} .
$$

Here $J_{0}$ is an assumed average ferromagnetic exchange, $\Delta J_{i j}$ represents the exchange fluctuations, $D$ an average local uniaxial anisotropy arising from electric field gradients of neighboring atoms, and $\hat{n}_{i}$ the random easy axis direction for the $i$ th spin. If $\Delta J_{i j}$ are taken to be zero, Eq. (l) becomes the Harris-Plischke-Zuckermann (HPZ) Hamiltonian on which much of the theory of the RMA problem is based. ${ }^{1}$ It is important to realize, however, that rare-earth glasses are alloys so that, in general, all three components of Eq. (1) will be required to consider a real material. Figure 1 illustrates this point in a schematic diagram in which $t \equiv k_{B} T / J_{0}$, $d \equiv D / J_{0}$, and $\delta \equiv\left\langle\Delta J_{>} / J_{0}\right.$. Clearly in the $d=0$ plane, one has the possibility for ferromagnetic (F), spin-glass (SG), and mixed $(M)$ phases, the latter of which refers to cases where there may be freezing of the longitudinal or transverse, or both, components of the spins. In the $\delta=0$ plane, the speromagnetic (SM) phase refers to a frozen, random magnetic structure with no long-range order; this structure is similar to that of a spin glass but we reserve the term for cases in which the origin of the spin scatter is RMA rather than frustration due to mixed interactions as in a spin glass. The correlated speromagnetic state (CSM), a new concept introduced by Chudnovsky and Serota, ${ }^{2}$ will be discussed further below. In investigating the CSM or SG states, it should always be borne in mind that one probably cannot have a material relevant to either the $t-d$ or $t-\delta$ planes. Thus, considerable caution is required when comparing data to theories developed for either case.

A high level of theoretical interest has been sustained in the RMA problem. Aharony used renormalization group techniques to study the critical behavior of the HPZ Hamiltonian. ${ }^{3} \mathrm{He}$ found for space dimensionality $d \leqslant 4$ no stable fixed point was approached and suggested that there is no sharp transition or that the transition is "smeared". He also stated that, "The exact meaning of this is not fully understood". Chen and Lubensky showed an important connection between the RMA and SG problems, viz., for sufficiently large $D$ the effective energy density has the same form as that of the random bond SG model with Ising symmetry. ${ }^{4}$ Pelcovits, Pytte, and Rudnick showed for $m>2$, there is no long-range ferromagnetic order in the RMA model for $d<4$, where $m$ is the number of spin components. ${ }^{5}$ Thus, the lower critical dimensionality is 4 .

Aharony and Pytte reported the existence of an infinite susceptibility phase in the small $D / J_{0}$ case and for $d<4$. $^{6}$ However, later work by the same authors concluded that the

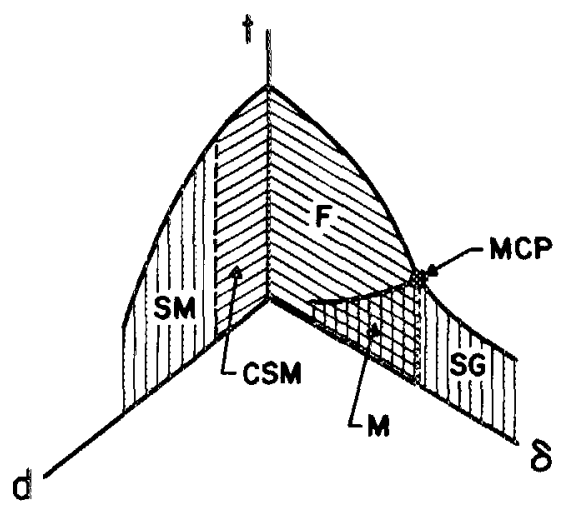

FIG. 1. Schematic phase diagram of possible magnetic states in the presence of RMA and exchange fluctuations (see text). 
susceptibility was limited for $T<T_{c}$ (Ref. 7$)$ to

$$
\chi_{\max } \propto\left(J_{0} / D\right)^{4} .
$$

This result has been verified by several subsequent calculations.

Chudnovsky and Serota (CS) recently have given a theory for the RMA problem in both the small and large $D / J_{0}$ limits. ${ }^{2}$ The magnetic structure is shown to depend strongly on the parameter $\lambda_{r} \equiv \lambda_{r}\left(R_{c} / a\right)^{2}$, where $\lambda_{r}=D / J_{0}, R_{c}$ is the spatial correlation of the easy axes, and $a$ is the atomic spacing. Of special interest is the prediction of the correlated spin glass, or in our convention, the correlated speromagnet. The CSM exhibits a smooth rotation of the magnetization $(M)$ over a ferromagnetic correlation length even though the net magnetization is zero. In the CSM, $M$ is reversible and there is a large zero-field susceptibility as in Eq. (2). The CSM is different from a multidomain ferromagnet in that $M$ rotates smoothly over the sample and there are no sharp domain walls. CS also find that the CSM state is sensitive to any uniform component of anisotropy or coherent anisotropy $D_{c}$. In fact, if $D_{c}$ is too large, the CSM state is converted into a ferromagnetic domain structure.

Additional theoretical predictions of CS concern the behavior of the magnetization in the CSM state. The authors show that, in reduced field units $(H), M(H)$ is given by

$$
\begin{aligned}
& M=1-(1 / 15) \Lambda_{r}^{2}\left(H+A_{c}\right)^{-1 / 2} \text { for } H<1, \\
& M=1-(1 / 15) \Lambda_{r}^{2}\left(H+A_{c}+1\right)^{-2} \text { for } H>1 .
\end{aligned}
$$

Eq. (4) with $\Lambda_{c} \equiv\left(D_{c} / J_{0}\right)\left(R_{c} / a\right)^{2}<1$ is similar to the result obtained by Callen $e t$ al..$^{8}$ which is valid also in the strong RMA case, i.e., $\Lambda_{r}>1$.

Finally, CS predict the existence of CSM like states in amorphous two-subnetwork magnetic glasses. For example, in an amorphous ferrimagnet, the addition of RMA should transform this state into a CSM state in which locally the magnetizations of the subnetworks are antiparallel but they rotate smoothly throughout the sample.

Recently, Dotsenko and Feigelman' studied the threedimensional planar magnet ( $X Y$ model) with random anisotropy fields for various orders of anisotropy, $n=1,2,3, \cdots$. $n=1$ and 2 correspond to the random field and random anisotropy cases, respectively. These authors calculate the zero-field susceptibility and find for the RMA case a cusplike behavior in $\chi(T)$. However, there is a region $\Delta T$ near $T_{c}$ which is outside the range of validity of the calculation. As $T \rightarrow T_{c}$, the authors find behavior that is "so far rather obscure," but no evidence for the infinite susceptibility phase was found. Goldschmidt recently has studied the behavior of the RMA problem in the large $N$ limit, where $N$ is the number of spin components. ${ }^{10}$ His results agree with $\mathrm{Eq} .(2)$ and, in addition, he determined the equation of state.

In summary, the theoretical situation for the RMA problem has seen several new predictions recently and we shall refer to these in the following sections of this paper. In addition, the character of the "transition" in the RMA case is obscure but there are indications that it may be related to the paramagnetic-spin-glass transition.

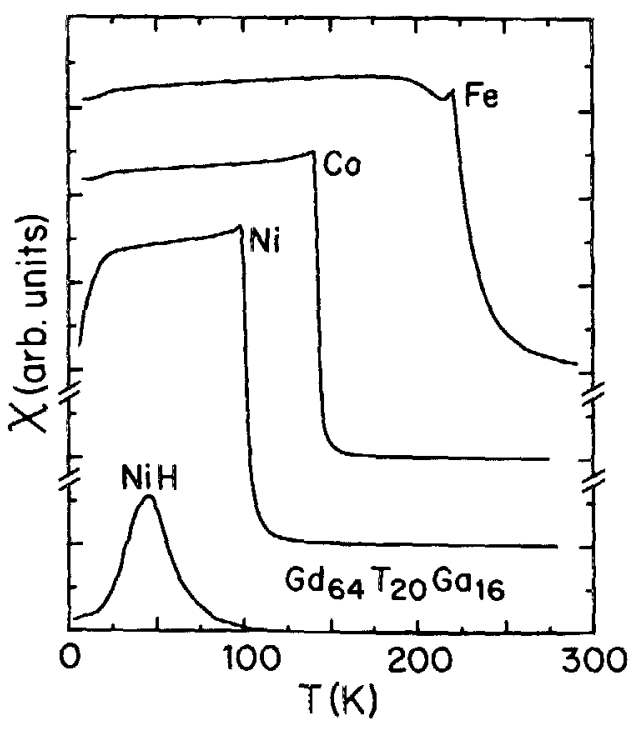

FIG. 2. ac susceptibility vs temperature for $\mathrm{Gd}_{64} \mathrm{~T}_{20} \mathrm{Ga}_{16}$ where $T=\mathrm{Fe}$, $\mathrm{Co}, \mathrm{Ni}$, and for $\mathrm{Gd}_{64} \mathrm{~T}_{20} \mathrm{Ga}_{16} \mathrm{H}_{120}$.

\section{EXPERIMENTAL RESULTS IN THE SMALL $D / J_{0}$ LIMIT}

Referring to Fig. 1, it is clear that observations of ferromagnetic or CSM states are most likely to occur in Gd-rich glasses. This follows since to first order there is no orbital angular momentum and thus no single-ion anisotropy. However, there could be a weak anisotropy due to a small admixture of higher $L$ states into the ground state. "If it is assumed for the moment that $D=0$, then one can probe the phase diagram in $t-\delta$ space. Figure 2 shows the ac susceptibility of several $\mathrm{Gd}_{64} \mathrm{~T}_{20} \mathrm{Ga}_{16}$ glasses, where $\mathrm{T}=\mathrm{Fe}, \mathrm{Co}$, Ni. These data were obtained at a frequency of $280 \mathrm{~Hz}$ and in RMS applied fields of about 0.1 Oe. ${ }^{12} \chi$ approaches the demagnetization limit $\left(N^{-1}\right)$ at temperatures $T_{c}$ which decrease as the strength of the exchange interactions decrease. In the $\mathrm{Ni}$ glass, the low temperature susceptibility decreases suggesting the entrance into a spin-glass phase. If $H$ is added to the Ni glass to the level of $[H] /[M]=1.2$, the average exchange is further diminished, with perhaps an increase in $\langle\Delta J\rangle$, and only a spin-glass like transition is observed. All of these data can be rationalized by the phase diagram of Fig. 1 in the $t-\delta$ plane. We have studied in detail two systems in which it was possible by alloying to more clearly delineate the ferromagnetic-spin-glass or reentrant transition. These systems were $\mathrm{Gd}_{x} \mathrm{La}_{72-x} \mathrm{G}_{28}$ and $\mathrm{Gd}_{x} \mathrm{La}_{65-x} \mathrm{G}_{35}^{\prime}$ where $\mathrm{G}_{25}$ and $\mathrm{G}_{35}^{\prime}$ represent $\mathrm{Ga}_{18} \mathrm{~B}_{10}$ and $\mathrm{Co}_{25} \mathrm{~B}_{10}$, respectively. In particular, in the former series, for the composition $\mathrm{Gd}_{72} \mathrm{G}_{28}$ and $\mathrm{Gd}_{68} \mathrm{La}_{4} \mathrm{G}_{28}$, a study has been made of the critical behavior at both $T_{c}$ and $T_{f g}$, where $T_{f g}$ denotes an assumed F-SG transition. In addition, the behavior of the isothermal remanent and thermoremanent magnetization was studied in the reentrant spin-glass phases. By performing a scaling analysis, it was possible to determine $\beta$ and $\delta$ values which gave good scaling at both transitions in both samples. These results are reported in detail elsewhere. ${ }^{13}$ However, it is of interest to point out here that, in general, for a given sample, the $\beta$ and $\delta$ values at $T_{c}$ and $T_{f 8}$ are not equal. This fact bears upon recent work of Binder who considered the static sus- 
ceptibilities of diluted ferromagnets such as $\mathrm{Eu}_{x} \mathrm{Sr}_{1-x} \mathrm{~S}^{11}$ In his theory, the P-SG line of Fig. 1 represents a glass transition, a dynamic nonequilibrium phenomenon, rather than an equilibrium phase transition. This implies the critical behavior should be essentially universal along both the upper (P-F) and lower (F-SG or F-M) lines in Fig. 1. As mentioned above, our critical exponents are not the same at $T_{c}$ and $T_{f g}$ which suggests that the idea of universality does not hold, at least for our GdLa glasses.

Another interesting problem concerning our Gd-rich glasses relates to the question: Could the apparent ferromagnetic like state seen below $T_{c}$ actually be the correlated speromagnetic state (CSM) predicted by Chudnovsky and Serota? Initially one might think not because we have shown that a scaling analysis was successfully performed at $T_{c}$ and this implies a diverging susceptibility for $T \rightarrow T_{c}^{+}-$a true ferromagnetic transition. On the affirmative side, however, is the fact that no spontaneous magnetization could be detected and the magnetization is completely reversible below $T_{c}$ (at least down to $T_{f g}$ ). It may be the case that our ac susceptibility measurements below $T_{c}$ indicate only that $\chi>N^{-1}$ so that $\chi_{\mathrm{ac}} \rightarrow N^{-1}$. This would be consistent with the theoretical prediction $\chi \sim\left(J_{0} / D\right)^{4}$, Eq. (4), which is very large for three samples. We have tested for $\mathrm{Gd}_{72} \mathrm{Fe}_{10} \mathrm{Ga}_{18}$, the field dependence of the magnetization in comparison to the CSM prediction of Eq. (2). Figure 3 shows the results of the fit, between $\sim 1$ and $80 \mathrm{kOe}$, to

$$
M=M_{0}\left[1-A H^{-1 / 2}\right]+\chi_{H F} H .
$$

Assuming that $A_{c}=0$ (there is no evidence in our samples for coherent anisotropy), the fit gives $M_{0}=220 \mathrm{emu} / \mathrm{g}$, $A=3.48(\mathrm{Oe})^{1 / 2}$, and $\chi_{H F}=38.9 \mu \mathrm{emu} / \mathrm{gOe}$. Over the field range of the fit, the $H^{-1 / 2}$ function gives a better fit than either an $H^{-1}$ or $H^{-2}$ function. The $M_{0}$ and $\chi_{H F}$ values are reasonable and $A$ can be used to calculate a $D$ value and an effective anistropy constant, $K=n D=7 \times 10^{6} \mathrm{erg} / \mathrm{cm}^{3}$. This value of $\mathrm{K}$ also is reasonable and is in rough agreement with earlier estimates of Cornelison and Sellmyer. ${ }^{12}$ Is the interpretation of the scaling analysis and ferromagnetic transition in these Gd-rich glasses consistent with the hypothesis that they also are examples of the CSM state? We suggest that the two are reconcilable if the reasonable as-

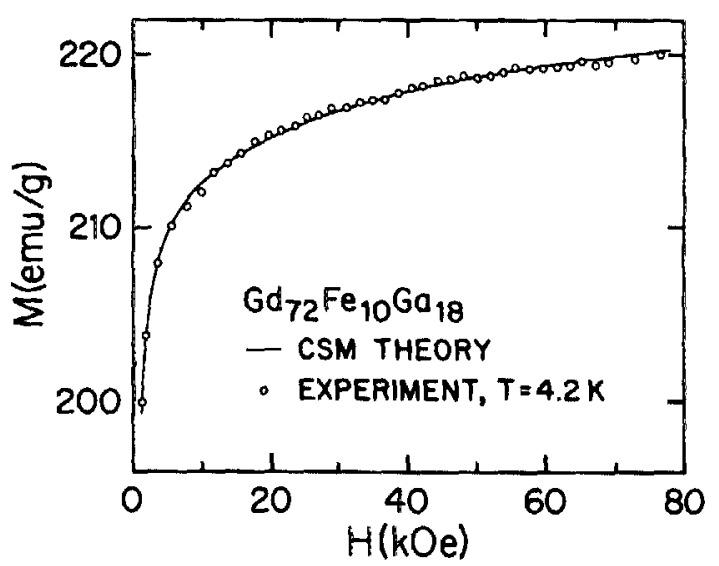

FIG. 3. Magnetization of $\mathrm{Gd}_{72} \mathrm{Fe}_{10} \mathrm{Ga}_{18}$ at $4.2 \mathrm{~K}$ and comparison to $\mathrm{CSM}$ prediction. sumption is made that in an applied dc field the P-CSM transition reverts to something like a standard P-F transition with appropriate critical exponents. In summary, if the apparent random anisotropy exhibited by our Gd-rich glasses can be represented as a single-ion anisotropy term like that of Eq. (1), then our data may be taken as evidence for the new CSM state predicted by Chudnovsky and Serota.

\section{EXPERIMENTAL RESULTS IN THE LARGE $D / J_{0}$ LIMIT}

Figure 4 shows the ac susceptibility of two glasses with large $D / J_{0}$ ratios: $\mathrm{Nd}_{64} \mathrm{Co}_{20} \mathrm{Ga}_{16}$ and $\mathrm{Tb}_{64} \mathrm{Co}_{20} \mathrm{Ga}_{16}$. These can be characterized as sharp speromagnetic transitions, especially the former where $\delta T / T_{f}=0.07, \delta T$ being the FWHM of the transition. Such transitions are much sharper than any of the canonical spin-glass transitions (e.g. Cu:Mn, $\mathrm{Au}: \mathrm{Fe}$ ) of which we are aware. This raises the interesting question of whether these transitions can be regarded as true phase transitions or, on the other hand, whether they have essentially the same character as spin-glass transitions. Although a consensus seems to have developed that the SG transition is a glass transition rather than a phase transition, there is evidence from the scaling analyses performed on $\mathrm{Cu}$ $\mathrm{Mn}^{14}$ and $a-\mathrm{GdAl}_{2}{ }^{15}$ that a phase transition picture is relevant. Also, some recent theoretical work suggests a true SG phase transition at finite temperature. ${ }^{16}$

The inset figure of Fig. 4 sketches the theoretical result mentioned earlier of Dotsenko and Feigelman. ${ }^{9}$ Again, the calculation breaks down near $T_{c}$ but there is some resemblance, at least, between the theory and our experimental results. A specific point of disagreement is that $\chi_{\text {expt }}(T \rightarrow 0)$

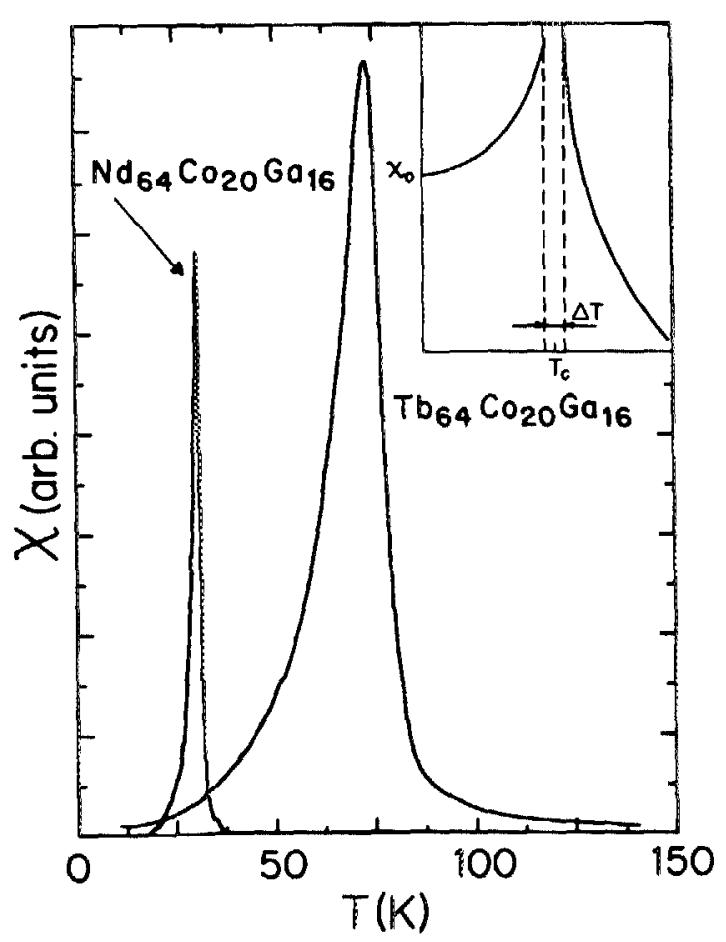

FIG. 4. ac susceptibility vs temperature for $\mathrm{Nd}_{64} \mathrm{CO}_{20} \mathrm{Ga}_{16}$ and $\mathrm{Tb}_{64} \mathrm{CO}_{20} \mathrm{Ga}_{16}$. The inset is an RMA calculation prediction of Dotsenko and Feigelman. 


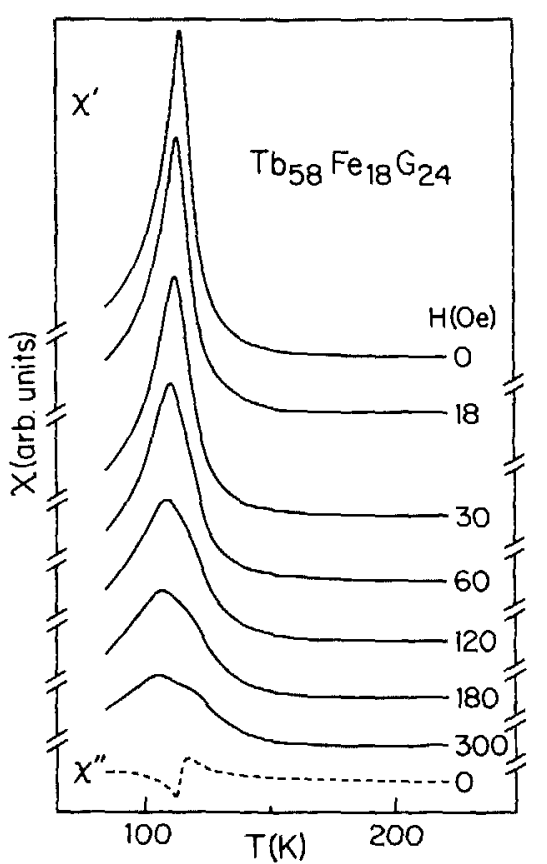

FIG. 5. Field-cooled ac susceptibility of $\mathrm{Tb}_{58} \mathrm{Fe}_{18} \mathrm{G}_{24}$ in various dc applied fields. The dashed curve is the out-of-phase component of $\chi$ for $H=0$.

approaches zero whereas $\chi_{\text {thy }}(T \rightarrow 0)$ approaches a constant.

Because of the relative sharpness of the speromagnetic transitions seen in rare-earth-rich glasses, we have studied the susceptibility behavior of $\mathrm{Tb}_{58} \mathrm{Fe}_{18} \mathrm{Al}_{14} \mathrm{~B}_{10}$, hereafter denoted as $\mathrm{Tb}_{58} \mathrm{Fe}_{18} \mathrm{G}_{24}$, in an applied field. This glass was chosen for detailed study for the technical reason that its transition temperature $\left(T_{f}\right)$ is in a more convenient region $(114.3 \mathrm{~K})$ than the glasses of Fig. 4 . One knows that Tb-rich glasses show field-cooling effects similar to those seen in spin glasses. ${ }^{12}$ Since $D / J_{0}$ is estimated to be about 5 for $\mathrm{Tb}_{58} \mathrm{Fe}_{18} \mathrm{G}_{24}$, we take seriously the idea mentioned earlier that RMA glasses can be considered as equivalent, in some sense, to Ising spin glasses. Following this approach, we show in Fig. 5 the in-phase component $\left(\chi^{\prime}\right)$ of the ac susceptibility for several values of dc field $(H)$ applied parallel to the ac field. The out-of-phase component $\left(\chi^{\prime \prime}\right)$ is shown only for $H=0$. In order to study the field dependence of $\chi$ we make the following definitions:

$$
\begin{aligned}
& \chi(H, T) \equiv\left[\chi^{\prime}(H, T)^{2}+\chi^{\prime \prime}(H, T)^{2}\right\}^{1 / 2}, \\
& \chi \equiv \chi_{0}-\chi_{H} .
\end{aligned}
$$

Here $\chi_{0}$ is the linear susceptibility for $H=0$ and $\chi_{H}$ the nonlinear susceptibility. For temperatures $T>T_{f}$ one can write $\chi_{H} \equiv \chi_{n l} H^{2}$ but as $T \rightarrow T_{f} \chi_{H}$ cannot be expanded in a power series in $H .{ }^{15}$ In general, the smallness of $\chi$ " makes it irrelevant whether $\chi_{0}$ and $\chi_{H}$ are determined from $\chi$ or $\chi^{\prime}$. It is interesting to note that for large values of $H$ (180 and 300 Oe), a distinct shoulder is seen in $\chi^{\prime}$ at $T>T_{\max } \equiv T_{f}$; the character of $\chi$ ' is reminiscent of the "double-transition" behavior seen in the GdLaG series. ${ }^{1,13}$

Figure 6 shows $\chi_{0}(T)$ and $\chi_{H}(T)$ for $H=60$ and 1800 e. It is seen that the nonlinear susceptibility has sharper peaks than $\chi_{0}$ but that the speromagnetic freezing at $T_{f}$ prevents both $\chi_{0}$ and $\chi_{H}$ from diverging. These curves are similar to

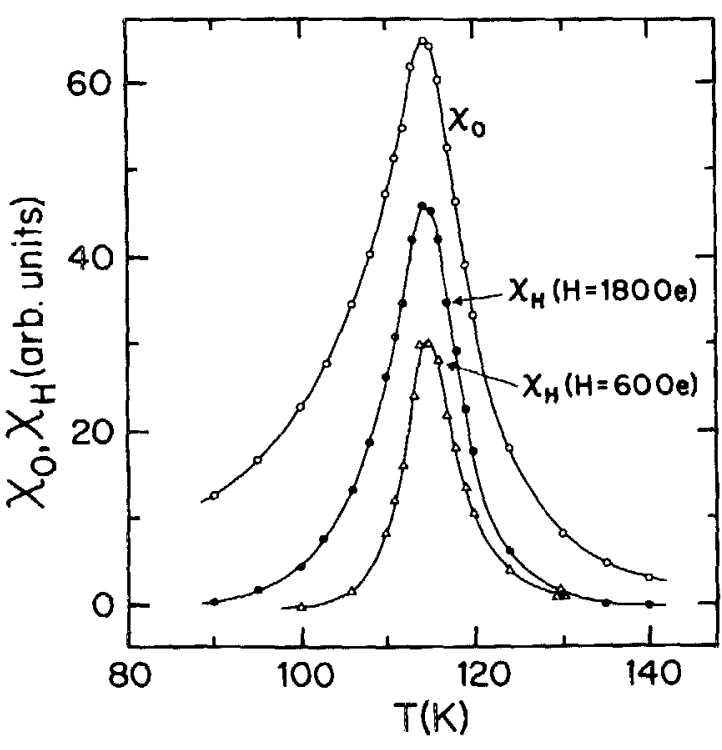

FIG. 6. Temperature dependence of $\chi_{0}$ and $\chi_{H}$ for $H=18$ and $60 \mathrm{Oe}$.

those calculated by Binder for the case where there is a competition between ferromagnetic and spin-glass type shortrange order. ${ }^{17}$ Above $T_{f}$, this system clearly exhibits ferromagnetic correlations which cause its susceptibility to become large. Following Binder, ${ }^{17}$ if we write

$$
\begin{aligned}
& \chi_{0}=C\left(T-T_{f}\right)^{-\gamma_{0}}+\chi_{1}, \\
& \chi_{H}=C\left(T-T_{f}\right)^{-\gamma_{H},}
\end{aligned}
$$

and restrict ourselves to temperatures not too close to $T_{f}$, we can determine $\gamma_{0}$ and $\gamma_{H}$ as shown in Fig. 7. The values obtained (2.4 and 2.3) certainly are larger than the meanfield value (1) but are somewhat smaller than those determined by Barbara and Malozemof ${ }^{15}$ for $a-\mathrm{GdAl}_{2}(2.7)$ and Omari ${ }^{14}$ et al. for Cu:Mn (2.75). At $T=T_{f}$, the scaling theory gives

$$
1-\frac{\chi}{\chi_{0}} \propto \chi_{H} \propto H^{2 / \delta},
$$

where $\delta$ is related to the other critical exponents by the scaling law $\delta=1+\gamma / \beta$. Figure 8 shows the field dependence of

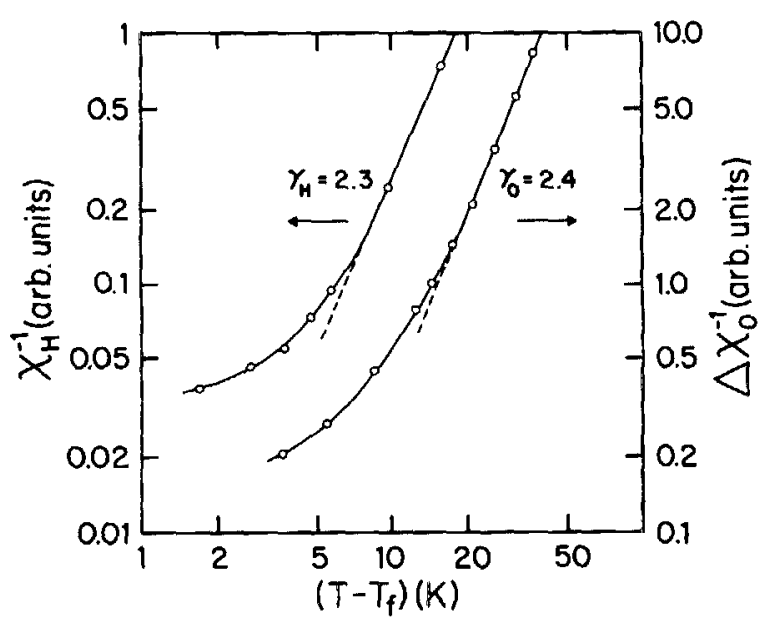

FIG. 7. $\log$ - $\log$ plots of $\Delta \chi_{0}^{-1}$ and $\chi{ }_{H}^{-1}$ vs $T$ to determine $\gamma$. [See Eqs. (8) and (9).] 


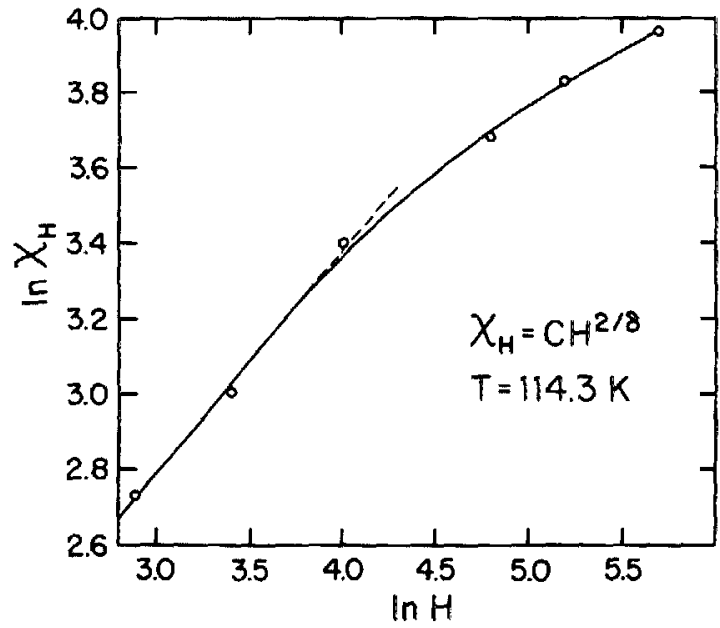

FIG. $8 . \ln \chi_{H}$ vs $\ln H$ for $T=114.3 \mathrm{~K}$.

$\chi_{H}$. In the low-field region $(H \leqslant 60 \mathrm{Oe}), \delta=3.6$. The falloff of $\chi_{H}$ at higher fields has been seen in spin glasses such as $\mathrm{Cu}: \mathrm{Mn}^{18}$ and $a-\mathrm{MnSi} .{ }^{19}$ From the scaling relation, $\beta=\gamma(\delta-1)^{-1}=0.9$. Although we have attempted to estimate $\beta$ by determining the temperature dependence of the order parameter $q(T) \propto\left(T_{f}-T\right)^{\beta}$, we do not trust the result because we are not certain how the ac measurement affects $\chi(T)$ below $T_{f}$, where viscous effects begin to appear. A second scaling law can be used to estimate the specific heat exponent: $\alpha=2-\beta(1+\delta)=-2.1$. This value is similar to that determined for $\alpha-\mathrm{GdAl}_{2}$ (Ref. 15) $(-2.7)$.

Finally, we have considered the phase diagram of speromagnetic $\mathrm{Tb}_{58} \mathrm{Fe}_{18} \mathrm{G}_{24}$ in a magnetic field. The equation $H=H_{0} \tau^{1.4}$ describes the field dependence of the assumed transition temperature, where $\tau \equiv 1-T / T_{f}$. It is interesting that this is similar to the de Almeida-Thouless ${ }^{20}$ fielddependent instability line in the mean-field model of an Ising spin glass: $H=H_{\mathrm{AT}} \tau^{3 / 2}$. Of course, we do not suggest that our system with its strong RMA and average ferromagnetic exchange is directly related to the AT theory. However, the Ising character of the RM.A system is suggestive.

\section{CONCLUSIONS}

In summary, we have discussed evidence for a lack of universality in Gd glasses showing double transitions. Certain of our Gd glasses also show behavior predicted for the CSM state by Chudnovsky and Serota. It was possible to analyze the field-dependent susceptibility of an RMA glass in terms of a scaling theory developed for spin glasses, relying on the similarity of the strong RMA problem to that of an Ising spin glass. It is our hope that these results will stimulate Monte Carlo or other theoretical work relevant to the case of RMA with a positive average exchange.

We are grateful to Dr. M. J. O'Shea, Dr. C. G. Robbins, Ms. Rebecca Richards, and Mr. G. McConnell for assistance and helpful discussions. The work was supported in part by the National Science Foundation under grant No. DMR-8110520.

'For a review and list of early references, see: D. J. Sellmyer and $M$. J. O'Shea, J. Less Comm. Metals 94, 59 (1984).

${ }^{2}$ E. M. Chudnovsky and R. A. Serota, J. Phys. C 16, 4181 (1983).

${ }^{3}$ A. Aharony, Phys. Rev. B 12, 1038 (1975).

${ }^{4}$ J. H. Chen and T. C. Lubensky, Phys. Rev. B. 16, 2106 (1977).

${ }^{5}$ R. A. Pelcovits, E. Pytte, J. Rudnick, Phys. Rev. Lett. 40, 476 (1978); 48, 1297 (1982).

${ }^{6} A$. Aharony and E. Pytte, Phys. Rev. Lett. 45, 1583 (1980).

${ }^{7}$ A. Aharony and E. Pytte, Phys. Rev. B 27, 5872 (1983).

${ }^{8}$ E. Callen, Y. Liu, and J. R. Cullen, Phys. Rev. B 16, 263 (1977).

${ }^{9}$ V. S. Dotsenko and M. V. Feigelman, J. Phys. C 16, L803 (1983).

10Y. Goldschmidt, Nucl. Phys. B 225 [FS9], 123 (1983).

"See: M. J. O'Shea, S. G. Cornelison, Z. D. Chen, and D. J. Sellmyer, Solid State Commun. 46, 313 (1983).

${ }^{12}$ For further details see: S. G. Cornelison and D. J. Sellmyer, Phys. Rev. B 30, 2845 (1984).

${ }^{13}$ M. J. O'Shea and D. J. Sellmyer, Bull. Am. Phys. Soc. 29, 336 (1984) (to be published).

${ }^{14}$ R. Omari, J. J. Prejean, and J. Souletie, J. Phys. 44, 1069 (1983).

${ }^{15}$ B. Barbara and A. P. Malozemoff, J. Less Commx. Metals 94, 45 (1983),

I6 See: A. J. Bray and M. A. Moore, J. Phys. C 17, L613 (1984); A. T. Ogielski and I. Morgenstern (these proceedings; A. P. Young (these proceedings); and references therein.

${ }^{17}$ K. Binder, Z. Phys. B 48, 319 (1982).

${ }^{18}$ C. Mulder, A. van Duyneveldt, and J. Mydosh, Phys. Rev. B 23, 1384 (1984).

19J. Hauser, F. Hsu, G. Kammlott, and J. Waszczak, Phys. Rev. B 20, 3391 (1974).

${ }^{20}$ J. R. L. de Almeida and D. J. Thouless, J. Phys. A 11, 983 (1978). 